\title{
La multimedia como herramienta para el aprendizaje autónomo del vocabulario del inglés por parte de los niños
}

\section{Multimedia as an Autonomous Learning Tool of English Vocabulary by Children}

Francisco Moreno Mosquera

Full time teacher, Programa de Inglés y Francés Universidad Tecnológica del Chocó Diego Luis Córdoba

Quibdó, Colombia

Email:framomo@hotmail.com

\begin{abstract}
This paper describes a pedagogical intervention carried out at the University of Chocó aimed at determining the degree of effectiveness of multimedia technology as a tool for independent learning of English vocabulary by children. The research problem was stated in terms of the questions: ¿how effective is foreign language learning through technology?, ¿how much can children in Chocó learn English vocabulary by themselves through multimedia? The findings confirm several hypotheses about the learning of vocabulary and pronunciation, and leads the researchers to conclude that multimedia is effective as a teaching tool for autonomous learning of foreign languages.

Keywords: multimedia, new technologies, computer, learning, English, children, vocabulary, pronunciation.

\section{Resumen}

Este artículo describe una Investigación pedagógica realizada en la Universidad del Chocó orientada a comprobar el grado de efectividad de la multimedia como herramienta para el aprendizaje autónomo del vocabulario del inglés por parte de los niños. El problema a investigar se planteó en términos de los interrogantes: ¿qué tan efectivo es el aprendizaje de una lengua extranjera a través de la tecnología?, ¿qué tanto pueden aprender los niños chocoanos por sí solos el vocabulario del inglés por medio de la multimedia? Los hallazgos encontrados confirman varias hipótesis sobre el aprendizaje del vocabulario y la pronunciación y permiten concluir que la multimedia es eficaz como herramienta didáctica para el aprendizaje autónomo de la lengua extranjera.
\end{abstract}

Palabras clave: multimedia, nuevas tecnologías, computador, aprendizaje, inglés, niños, vocabulario, pronunciación. 


\section{Introducción}

En el ámbito mundial se han realizado innumerables investigaciones que evidencian las bondades de la incorporación de las nuevas tecnologías en la enseñanza de lenguas extranjeras. En Colombia, si bien el país apenas comienza a incursionar en la corriente mundial de apropiación y uso de la tecnología en educación, el tema ha despertado la atención de la comunidad académica nacional, especialmente en algunas universidades como la Universidad de la Sabana y la Universidad Distrital Francisco José de Caldas de Bogotá, en cuyos programas de Maestría se han venido desarrollando una serie de estudios los cuales han hecho aportes que, de una u otra forma, están contribuyendo a configurar un panorama sobre la utilización de la tecnología como mediación pedagógica. Por eso, con el objeto de entender y analizar los efectos de estas nuevas herramientas didácticas en nuestro propio terreno, en el año 2008 el Grupo de Investigación en Didáctica de los Idiomas Extranjeros de la Universidad Tecnológica del Chocó desarrolló una intervención pedagógica orientada por las siguientes preguntas ¿Qué tan efectivo es el aprendizaje de una lengua extranjera a través de la tecnología? ¿Qué tanto pueden aprender los niños chocoanos por sí solos el vocabulario del inglés por medio de la multimedia?

Se abordó el tema con el propósito de verificar las diversas tesis que favorecen el uso de la tecnología multimedia en la enseñanza de idiomas extranjeros e igualmente con el fin de motivar a los docentes y crear conciencia en Colombia sobre la importancia de innovar las prácticas tradicionales de enseñanza del inglés mediante la incorporación de la tecnología, como aliada del proceso didáctico en las instituciones educativas.

\section{Referentes teóricos}

Dado que esta investigación involucra esencialmente tres asuntos, en primer lugar el aprendizaje de la lengua mediado por computador, en segundo lugar el aprendizaje del aspecto lexical del inglés, y en tercer lugar los niños como aprendices, para enmarcar el estudio dentro de un contexto teórico pertinente, se presentan a continuación algunas consideraciones sobre los siguientes temas, estrechamente relacionados con el objeto de la investigación:

- Tecnologías de información y la comunicación y teorías de aprendizaje de la lengua

- Aprendizaje de lenguas asistido por computador (CALL)

- La multimedia como medio para propiciar el aprendizaje

- El aprendizaje de vocabulario

- Aprendizaje de la lengua extranjera a temprana edad: hipótesis del período crítico

Tecnologías de información y la comunicación y teorías de aprendizaje de la lengua

A lo largo de la historia de la Humanidad se han propuesto diversas teorías sobre el aprendizaje humano, tratando de explicar cómo el hombre alcanza a desarrollar el lenguaje. A partir de la década del 60 se han formulado una variedad de hipótesis para explicar cómo se aprende un idioma y, con fundamento en tales hipótesis, han surgido diversos métodos y prácticas de enseñanza. Desde la Psicología se han distinguido esencialmente tres enfoques: el conductista, el cognitivista y el funcional.

Para el conductismo, la mente humana se comporta como una "caja negra" en la que el desarrollo cognitivo se da a través de la conducta. Esta visión se apoya en los trabajos de Skinner, quien a su vez desarrolla las tesis de Pavlov, en los que la relación estímulo-respuesta determina el proceso de aprendizaje. Este enfoque da origen a métodos de enseñanza como el Total Physical Response (TPR), cuyo principal exponente es Asher, y el método audiolingual, que tiene como premisa que la lengua se aprende por un proceso de imitación y, en consonancia, abundan en ejercicios de repetición oral.

Dentro del enfoque cognitivista se distinguen varias propuestas teóricas, entre las que se des- 
tacan el constructivismo de Bruner y Piaget y el innatismo propuesto por Chomsky, que plantea la existencia de un dispositivo de adquisición de la lengua ("Language Acquisition Device, LAD) con el cual nace el ser humano y que explica por qué el niño es capaz de asimilar y desarrollar un sistema tan complejo como es la lengua en tan corto tiempo.

Para el enfoque funcional el aprendiz va desarrollando la lengua a partir de la necesidad de interactuar significativamente y por lo tanto va adquiriendo las estructuras en la medida que éstas tienen significado para sus necesidades de comunicación.

En épocas recientes, a partir del advenimiento de las denominadas Tecnologías de la Información y las Comunicaciones (TICs) y su incidencia en la manera como el hombre del siglo XXI accede al conocimiento y desarrolla nuevas formas de aprendizaje, ha surgido la teoría del conectivismo, la cual se fundamenta en las limitaciones de las teorías conductista, cognitivista y constructivista frente a los efectos de la tecnología sobre la forma en que actualmente vivimos, nos comunicamos y aprendemos.

La teoría conectivista, propuesta en la presente década por George Siemens (2004), establece como premisa que el conocimiento no sólo reside dentro del ser humano sino también en otras fuentes (como por ejemplo máquinas y dispositivos electrónicos), por lo que es necesario nutrir y mantener conexiones para facilitar el aprendizaje continuo; el aprendizaje implica un proceso de conectar nodos o fuentes de información. Igualmente se asume que la capacidad para aumentar el conocimiento es más importante que lo que ya se sabe y que la habilidad para ver las conexiones entre los campos, ideas y conceptos es primordial.

El aprendizaje (definido como conocimiento aplicable) puede residir fuera de nosotros (al interior de una organización o una base de datos), está enfocado en conectar conjuntos de información especializada, y las conexiones que nos permiten aprender más tienen mayor importancia que nuestro estado actual de conocimiento (Siemens, 2004: 6).

El conectivismo es, en suma, una teoría de aprendizaje para la era digital en la cual interviene la tecnología como un elemento fundamental en el desarrollo cognitivo. Existe una estrecha relación entre esta teoría y el concepto de e-learning, o aprendizaje electrónico, en donde se dan ambientes propicios para el aprendizaje de la lengua mediado por los computadores, conocido como Computer Assisted Language Learning (CALL).

\section{Aprendizaje de lenguas asistido por computador (CALL)}

Hasta hace unas tres décadas era impensable que alguien pudiera aprender un idioma extranjero sin la intervención de un profesor. Era inconcebible la idea de un alumno sentado frente a una máquina, interactuando con ésta de manera oral y escrita, recibiendo de ella no sólo instrucciones sino también retroalimentación sobre su ejecución lingüística. Hoy en día, gracias a los formidables avances de las TICs, a los desarrollos de la multimedia y a la amplia penetración de la Internet, el rol de los computadores en el aprendizaje de lenguas extranjeras ha adquirido enorme importancia, reconocida por la casi totalidad de profesores en el mundo entero.

Con la puesta de la tecnología al servicio de la educación la enseñanza de lenguas extranjeras ha dado un salto cualitativo gigantesco que les ha significado a profesores y alumnos un cambio de las herramientas didácticas tradicionales por otras nuevas que facilitan el proceso.

\section{La multimedia como medio para propiciar el aprendizaje de la lengua}

La multimedia es, hoy por hoy, una herramienta didáctica con potencialidad y uso por parte de los desarrolladores de software educativo. Esta tecnología pone a disposición de 
profesores y alumnos una amplia variedad de recursos de incalculable valor para el aprendizaje de las lenguas. Un curso multimedia, al integrar medios diversos como texto, imágenes, audio, video, y animaciones, sumado a ello los sistemas de reconocimiento de voz, ofrece la posibilidad de un aprendizaje más eficaz y autónomo, ya que el alumno tiene el control y puede trabajar a su propio ritmo e imponer sus propios tiempos. Igualmente importante es el elemento lúdico, pues el sólo hecho de introducir el computador en la clase es motivador para la mayoría de estudiantes, a más de la gran variedad de recursos audio visuales que hacen a los cursos multimedia atractivos y divertidos.

Al hablar de las potencialidades de este recurso tecnológico, no podemos dejar de lado los conceptos de hipermedia e hipertexto que permiten al aprendiz leer un texto en múltiples direcciones y así mismo escoger rutas de lectura, de acuerdo con sus propios intereses. Dado que la hipermedia involucra una serie de interconexiones entre bloques o fragmentos de información (textual, pictórica, auditiva) que posibilitan abordar un texto de manera no secuencial sino multidireccional, la teoría que subyace a la hipermedia es que ésta se asemeja a la forma como opera el pensamiento humano.

El diseño de una estructura de bifurcaciones interconectadas es una de las peculiaridades de los hipermedia y es similar a los senderos ramificados del pensamiento humano; entramados arborescentes por los cuales es posible avanzar desde un principio único hasta un final múltiple. (Laura Regil).

Aunque el carácter hipermedial de la Internet le confiere un enorme potencial a la Red como recurso para la enseñanza de un idioma extranjero, es menester ser cautelosos en su utilización, entre otras razones dada la falta de garantías en cuanto a la calidad de lo que se ofrece en Internet, no sólo desde el punto de vista pedagógico sino también desde la perspectiva de la veracidad o autenticidad del material que se encuentra en la Red.

En todo caso las ventajas de la multimedia, sobre cualquier otro recurso son obvias, cuando se trata de la enseñanza de aspectos específicos como el vocabulario, entre otras razones porque se puede tener control sobre la muestra lingüística que se le va a presentar al alumno, al tiempo que se prescinde de elementos innecesarios que pueden desviar el foco de atención o que, en el peor de los casos, podrían agregar grados de dificultad al punto que se pretende enseñar.

Como se dijo atrás, la evolución de las TICs ha corrido en forma paralela a la de los métodos de enseñanza, y actualmente la multimedia ha llegado a ser un recurso idóneo para la implementación de aquellos enfoques modernos centrados en el alumno, quien ahora tiene mayor protagonismo y control sobre su proceso de aprendizaje.

Es pertinente resaltar algunas de las ventajas que ofrece la tecnología multimedia como recurso para la enseñanza de una lengua extranjera, en nuestro caso el inglés. Uno de los factores positivos es que se hace posible la individualización de la enseñanza, atendiendo los diversos estilos de aprendizaje. Con un curso multimedia el alumno tiene a su profesor disponible las 24 horas del día, 7 días a la semana; con un simple movimiento del dedo, al hacer click, puede escuchar la pronunciación de la lengua extranjera cuantas veces lo desee e igualmente, dada la interactividad de la tecnología, tiene la posibilidad de corregir su propia pronunciación.

Como efecto de lo anterior, otra de las ventajas evidentes de la multimedia es que le ahorra al docente tiempo y esfuerzos, por ejemplo modelando y repitiendo la pronunciación, ya que el computador puede hacer esta tarea por él. La tecnología facilita el seguimiento de los alumnos, lo que permite al docente monitorear el progreso individual de cada uno: cuáles y cuántas activi- 
dades ha llevado a cabo, su duración, número de ejercicios correctos e incorrectos, etc.

Un aspecto altamente positivo del uso de la multimedia tiene con ver con factores psicológicos ya que, al prestarse para el trabajo individual, constituye el recurso de aprendizaje ideal para aquellos alumnos que son extremadamente tímidos y que se sienten inhibidos para hablar en presencia del profesor o frente a sus compañeros de clase por temor a ser objeto de burlas. Siendo la autoestima y el filtro afectivo, en términos de Krashen (1981), uno de los factores que inciden en el aprendizaje de una lengua extranjera, es de suma importancia que al alumno se le posibilite practicar el idioma en un ambiente libre de ansiedades y de situaciones que puedan resultar perturbadoras.

En el marco de esta discusión cabe considerar los nuevos roles que las tecnologías imponen sobre profesores y estudiantes de la lengua extranjera. La revolución tecnológica de los últimos tiempos y su incorporación en el ámbito de la enseñanza de idiomas, plantea grandes retos que no siempre los docentes están dispuestos o en condiciones de asumir. El perfil del docente de la era digital comprende un profesional que no sólo sea hábil en el manejo de los computadores y uso de los programas informáticos, sino que también sea un verdadero facilitador del proceso de aprendizaje. Hoy en día se espera que el profesor ejerza un papel de guía del alumno, capaz de seleccionar, adaptar, evaluar, e incluso elaborar, material que facilite el aprendizaje de la lengua de acuerdo con las circunstancias específicas y los intereses de los alumnos.

Por su parte, en este nuevo paradigma de enseñanza, los aprendices del idioma dejan el papel tradicional de ser receptores pasivos, dependientes únicamente de lo que el profesor les presenta, para convertirse en actores y promotores de su proceso de aprendizaje. El estudiante moderno encuentra en las TICs la oportunidad de buscar otras fuentes e interactuar con otros modelos de la lengua diferentes a su profesor. La mediación tecnológica a través de la multimedia valida la teoría cognitiva y propicia, así, la aplicación de los enfoques constructivistas, que asumen el papel del alumno como alguien que participa activamente en la construcción de su propio conocimiento.

\section{El aprendizaje del vocabulario}

La enseñanza y el aprendizaje de vocabulario han sido durante largos años los aspectos que menor atención han recibido en los cursos de idiomas extranjeros, quizás debido a la creencia errónea de que lo más importante es el dominio estructural de la lengua, relegando el aspecto lexical a un segundo plano. Usualmente los cursos omiten la enseñanza directa de vocabulario, dejando que su aprendizaje se dé por cuenta del uso del diccionario y por procesos de inferencia. Hoy se sabe que a medida que el estudiante adquiere un mayor repertorio de palabras, éste gana confianza a la hora de leer o escribir en la lengua extranjera y que una riqueza de vocabulario no sólo le facilita al alumno comprender gran variedad de discursos sino que también le ayuda a identificar más fácilmente las estructuras gramaticales.

Abordando el aprendizaje de vocabulario de la lengua extranjera desde una perspectiva psicolingüística, los estudios de Henning (1973) arrojan algunas luces sobre la manera como el aprendiz procesa las palabras en su mente. Henning descubrió que, en las etapas tempranas del aprendizaje, el niño interioriza el vocabulario apoyándose en vínculos fonéticos, es decir, las palabras que tienen sonidos parecidos (como por ejemplo "night" y "light") son almacenadas dentro de un mismo "compartimento" en la memoria. De acuerdo con estos estudios, en etapas más avanzadas del aprendizaje la fijación del vocabulario se da con base en vínculos semánticos: se demostró que hay mayor facilidad de aprendizaje cuando al alumno se le enseñan juntas las palabras que tienen relación a nivel de significado o 
que pertenecen a un mismo campo semántico, tales como "father", "mother", "brother", "sister".

Desde el punto de vista lingüístico, el aprendizaje de vocabulario involucra aspectos semánticos, ortográficos y fonéticos. Se puede decir que un estudiante de inglés ha aprendido una palabra cuando, además de saber su significado, es capaz de escribirla y pronunciarla correctamente. Sin embargo hay que distinguir entre el conocimiento receptivo y el dominio activo que se posee del vocabulario: el primero implica que el aprendiz esté en capacidad de reconocer una determinada palabra al escucharla o leerla, aunque no pueda él mismo pronunciarla o escribirla, mientras que el domino activo abarca la comprensión y producción escrita y oral.

Al respecto, Steinberg (1996) y Radford (1999) y otros sostienen que el niño posee una capacidad innata para percibir y entender correctamente los sonidos de la lengua, la cual es superior a su capacidad para producir dichos sonidos; en otras palabras, el conocimiento receptivo es mayor al dominio activo.

La pronunciación es, pues, un aspecto central e inherente al aprendizaje de vocabulario en la lengua inglesa. Debido al carácter no fonológico de su escritura (a diferencia del español), el inglés presenta una dificultad obvia para el alumno hispanohablante a la hora de abordar la pronunciación de las palabras ya que ella no se puede predecir a partir de la forma escrita; por consiguiente, el aprendizaje de vocabulario debe ir de la mano con el aprendizaje de pronunciación.

\section{Aprendizaje de la lengua extranjera a} temprana edad: hipótesis del período crítico

No hay duda de que los primeros años de vida son la etapa ideal para la adquisición de la lengua, sea ésta la lengua nativa o un segundo idioma. Lenneberg (1967) propuso en la década del 60 la hipótesis del período crítico, que aún sigue siendo aceptada, según la cual desde el nacimiento hasta la pubertad existe una mayor disposición y capacidad cerebral para el desarrollo de las habilidades lingüísticas. Pasada la pubertad la capacidad para aprender la lengua sufre una disminución a medida que el cerebro va perdiendo plasticidad.

La hipótesis del período crítico se fundamenta en factores neurofisiológicos, entre los cuales juega un papel importante el fenómeno de la lateralización cerebral, relacionado con los hemisferios del cerebro. Por los desarrollos de la neurolingüística en este campo, hoy sabemos que cada zona del cerebro se especializa en determinadas funciones y que el procesamiento del lenguaje se produce principalmente en el hemisferio izquierdo, aunque el hemisferio derecho interviene para el procesamiento de la pronunciación y la entonación. Se cree que al llegar a la pubertad estas zonas cerebrales pierden su potencial de procesamiento y con mayor rapidez, el relacionado con los sonidos lingüísticos. Esto explica por qué -como lo destacan Bongaerts y otros (1997)- los niños tienen ventajas sobre los adultos a la hora de aprender a pronunciar una segunda lengua, es mucho más fácil para el niño alcanzar un nivel fonético cercano o idéntico al del hablante nativo.

Por otra parte también hay que considerar factores socio-afectivos. Generalmente, los niños no están mentalmente bloqueados por los prejuicios que muestran los adultos cuando tienen que aprender un nuevo sistema de comunicación, especialmente en el nivel fonético. Mientras los adultos se inhiben por temor a hacer el ridículo a la hora de pronunciar la lengua extranjera, los menores muestran una actitud menos negativa y no temen cometer errores, lo que facilita el proceso de aprendizaje.

Las cuestiones derivadas de la hipótesis del período crítico tienen una incidencia importante en la didáctica del idioma y deben ser tenidas en cuenta al momento de tomar decisiones en relación con la edad en que se debe empezar un programa de enseñanza de la lengua extranjera y 
con el enfoque pedagógico a seguir, dependiendo de la edad del aprendiz.

\section{Procedimientos metodológicos}

Se trata de una intervención pedagógica que se desarrolló en la ciudad de Quibdó, en las instalaciones de la Universidad Tecnológica del Chocó (UTCH) con un grupo de 10 niños (5 niños y 5 niñas), alumnos de primaria, con edades comprendidas entre los 6 y los 10 años, de estrato socio-económico medio, quienes viven en un barrio marginal de la ciudad y estudian en escuelas anexas a la UTCH. Los criterios para la selección de la muestra, a más del factor edad, fueron que el número de niños no fuera superior a 10, para facilitar su control.

A manera informativa se realizó una charla con los padres de familia y los maestros en la que se comprobó que los niños no habían tenido contacto alguno con la lengua extranjera y por ende, no conocían el vocabulario que se les iba a presentar en la intervención pedagógica. Dentro del laboratorio de idiomas de la Universidad Tecnológica del Chocó se acondicionó un espacio especial para ellos, con 10 computadores multimedia.

A los niños se les administró un programa multi-media sobre vocabulario, que comprendía un total de 36 palabras, agrupadas por temas: wild animals, domestic animals, household objects. Vale la pena resaltar que el aplicativo multimedia fue elaborado por una joven investigadora, egresada de la $\mathrm{UTCH}$, vinculada al equipo de investigación con el respaldo de Colciencias, quien jugó un papel importante en las diferentes etapas del estudio. Utilizando PowerPoint, imágenes descargadas de internet y voces nativas pregrabadas, la joven investigadora produjo un atractivo programa que contenía 12 palabras por área, organizadas en 4 páginas o diapositivas para cada tema.

\section{Intervención pedagógica}

El equipo de docentes-investigadores quiso darle a esta implementación pedagógica una dimensión social y formativa adicional a la experimentación científica, puesto que es un hecho reconocido que el departamento del Chocó, en el contexto colombiano, se encuentra a la zaga en materia de desarrollo socioeconómico y educativo, especialmente en lo relacionado con la alfabetización digital de los estudiantes. Teniendo en mente la necesidad de nuestros alumnos y docentes de aprender a manejar las herramientas tecnológicas, además de buscar respuestas a las preguntas mencionadas, se tenía el propósito de dejarles a los niños -que nunca antes habían tenido la experiencia de sentarse frente a un computador- una apropiación y un aprendizaje práctico en manejo de tecnología, como subproducto del estudio. Igualmente se le brindó a la joven docente, que participó con el equipo, la oportunidad de desarrollar habilidades para la observación y reflexión pedagógica e investigativa con los estudiantes. La intervención pedagógica se desarrolló en tres etapas: preparación, auto aprendizaje y evaluación.

\section{Etapa de preparación}

Se trató de una fase de aprestamiento. Durante una semana, con el acompañamiento de la joven investigadora, se familiarizó a los niños con el computador, se les enseñó el manejo del equipo y de la herramienta de enseñanza en PowerPoint. Fue sorprendente el hecho de que los niños no sólo aprendieron a navegar por el curso sino que también rápidamente adquirieron destrezas en la manipulación del computador y de algunas aplicaciones que se encontraban disponibles en el escritorio.

\section{Etapa de auto aprendizaje}

Una vez adquirieron las suficientes destrezas técnicas, los niños debían acudir a la universidad 
por tres días a la semana (lunes, miércoles y viernes) durante los cuales estudiaban el vocabulario, consistente en el texto escrito de las palabras, acompañado de imágenes y sonidos. Considerando su corta edad, cada jornada de trabajo tenía una duración máxima de 30 minutos. Esta etapa se desarrolló durante 6 semanas hasta completar 10 horas. Durante este tiempo los niños trabajaron por sí solos, a su propio ritmo y sin ninguna mediación docente en el proceso de aprendizaje del material. El papel de los docentes-investigadores solamente se limitó a darles asistencia a los niños en cuanto al manejo del computador o la manipulación de las herramientas del programa. El trabajo de los niños consistía, básicamente, en observar el texto de las palabras, mirar las imágenes, escuchar el audio e imitar la pronunciación. Al finalizar cada jornada se realizaban actividades lúdicas.

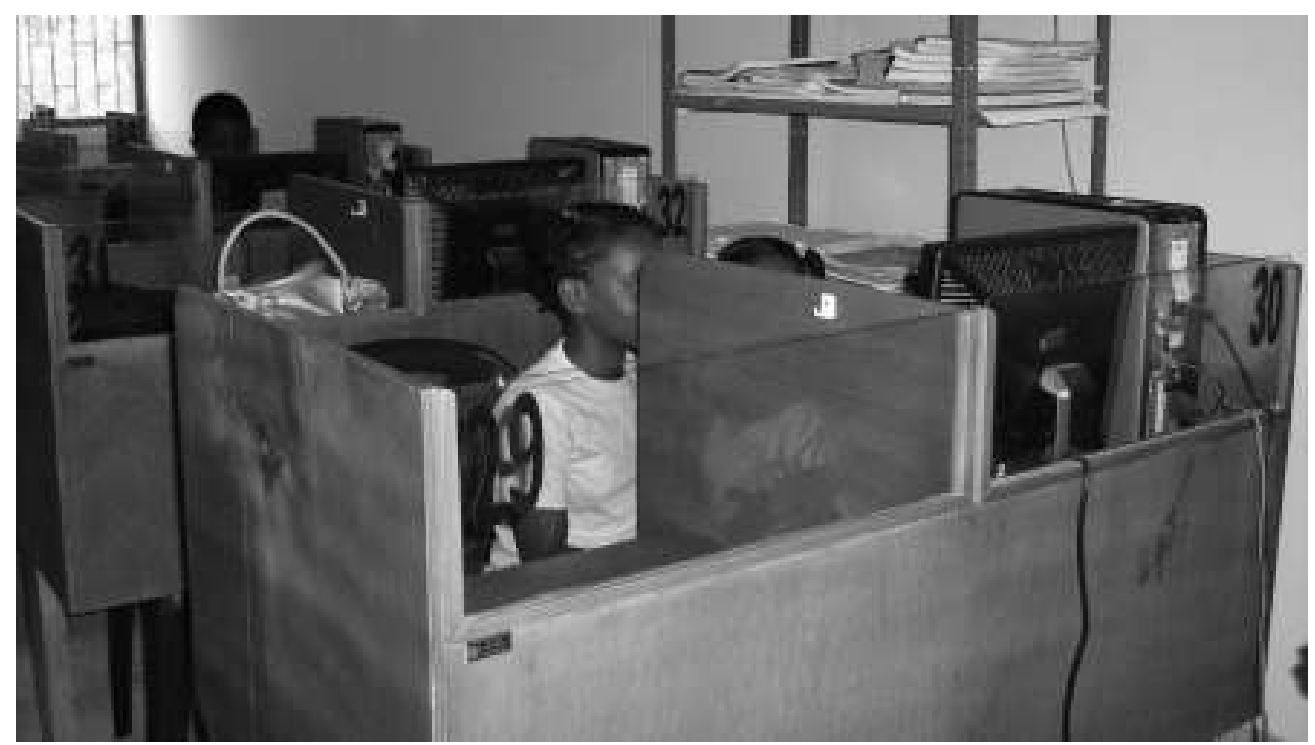

Niños del estudio trabajando en el laboratorio de idiomas de la Universidad Tecnológica del Chocó

\section{Etapa de evaluación}

Al final de la etapa de auto aprendizaje se les hizo una evaluación para ver el porcentaje del vocabulario aprendido, tanto a nivel receptivo (escucha) como productivo (pronunciación). Como instrumento de evaluación de la capacidad receptiva se utilizó un test de escucha e identificación; para evaluar la capacidad productiva, el niño debía pronunciar cada palabra, a partir de la imagen acompañada del texto.

\section{Hallazgos}

Un $77.8 \%$ del vocabulario objeto de estudio fue correctamente identificado por los niños a partir de la escucha, mientras que el $67.4 \%$ de las palabras fueron pronunciadas correctamente. Este resultado confirma que la habilidad receptiva es superior a la productiva. Cabe anotar que, aunque no es posible documentar en este reporte los registros de las voces infantiles, el nivel fonético alcanzado por los niños fue sorprendente ya que su pronunciación fue similar al de un hablante nativo del inglés. 
Porcentaje global de palabras aprendidas a nivel de escucha y de pronunciación

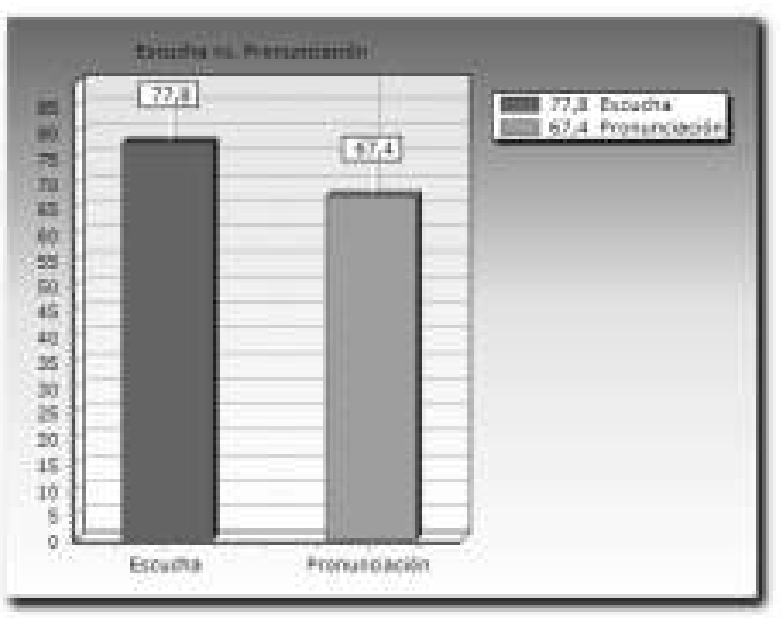

Estos resultados nos muestran, en primer lugar, que hubo un alto nivel de aprendizaje: el niño de mejor desempeño logró reconocer el 97\% de las palabras que escuchó y pudo pronunciar correctamente el $94 \%$ del vocabulario. En segundo lugar, se evidencia que en todos los casos la capacidad receptiva superó a la productiva; dicho en sentido contrario, ningún niño pudo pronunciar correctamente un mayor número de palabras de las que estuvo en capacidad de reconocer. De otro lado, al comparar los resultados individuales, se observa consistencia en los niveles de desempeño, conservándose la escala de valores en el balance escucha vs. pronunciación; es decir, no se presentó ningún caso en que un niño de calificación inferior en pronunciación, hubiera superado a nivel de escucha a otro niño con una calificación más alta que la suya en pronunciación.

Para ponderar la efectividad global del experimento, se promediaron las calificaciones de escucha y pronunciación obtenidas por todos los niños y se estableció el $60 \%$ de respuestas acertadas como valor satisfactorio. La tabla de arriba muestra que el $70 \%$ de los niños cruzaron este umbral.

\section{Porcentaje individual de palabras aprendidas a} nivel de escucha y de pronunciación

\begin{tabular}{|l|c|c|}
\hline \multicolumn{1}{|c|}{ NIÑO (A) } & ESCUCHA & PRONUNCIACIÓN \\
\hline No. 1 & $97 \%$ & $94 \%$ \\
\hline No. 2 & $91 \%$ & $77 \%$ \\
\hline No. 3 & $88 \%$ & $69 \%$ \\
\hline No. 4 & $83 \%$ & $75 \%$ \\
\hline No. 5 & $80 \%$ & $69 \%$ \\
\hline No. 6 & $80 \%$ & $69 \%$ \\
\hline No. & $77 \%$ & $75 \%$ \\
\hline No. 8 & $66 \%$ & $52 \%$ \\
\hline No. 9 & $58 \%$ & $42 \%$ \\
\hline No. 10 & $55 \%$ & $44 \%$ \\
\hline
\end{tabular}

Hallazgos en relación con las palabras individuales del vocabulario

\section{Pronunciación}

- Hubo sólo 3 palabras que todos los niños pronunciaron en forma correcta: Computer, Fish, Glass.

- La palabra que mayor cantidad de niños pronunció de manera incorrecta fue Picture (90\% de los niños), seguida de Knife (80\%), Fan y Table $(70 \% \mathrm{c} / \mathrm{u})$.

- Según estos datos, "computer", "fish" y "glass" se erigen como las palabras más fáciles de pronunciar, dentro de la muestra, en tanto que la pronunciación de la palabra "picture" presenta un alto grado de dificultad para los niños.

\section{Escucha}

- Se dieron 10 palabras que todos los niños pudieron reconocer al escucharlas: Computer, Television, Radio, Spoon, Glass, Mouse, Giraffe, Tiger, Elephant, Snake.

- La palabra que no pudo ser reconocida por la mayor cantidad de niños fue Bird (60\% de los niños), seguida de House, Door, Iron, Table, Fan, Dog (50\%, c/u). 
Porcentaje de niños que cruzaron el umbral

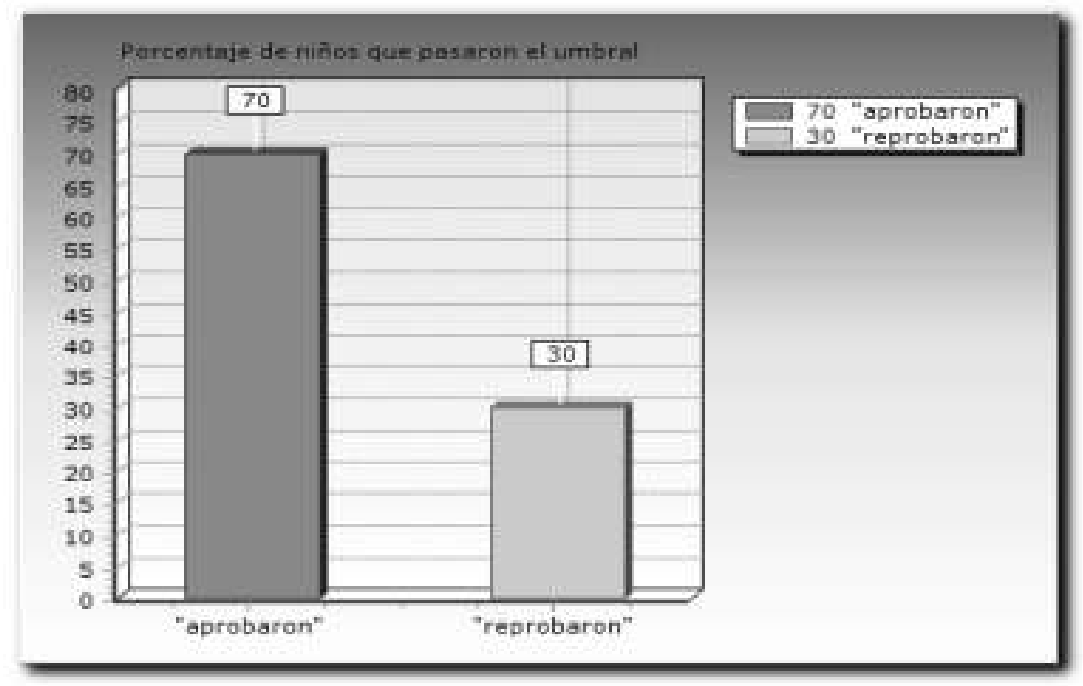

De nuevo aparece computer como un término de fácil aprendizaje: de la totalidad del vocabulario, ésta fue la palabra más fácil de adquirir tanto a nivel receptivo como productivo.

\section{Conclusiones}

Los hallazgos y resultados de esta interpretación permiten concluir, en primer lugar, que la multimedia es una herramienta efectiva para el aprendizaje autónomo del vocabulario del idioma extranjero. En segundo lugar, que en el aprendizaje del idioma las habilidades receptivas (en este caso la escucha) se desarrollan más que las productivas, es decir, el estudiante está en mayor capacidad de reconocer más palabras de las que puede pronunciar. Las TICs constituyen, pues, un recurso didáctico eficaz que ofrece valores agregados como el hecho de que los niños se sienten altamente motivados por esta nueva forma de aprender. Los docentes deberían tomar conciencia de la importancia de incorporar los recursos tecnológicos en la enseñanza ya que éstos, aunque no reemplazan al profesor, sí son un poderoso aliado en el proceso de enseñanza y aprendizaje.

\section{Referencias}

Bongaerts, T. et al. (1997). Age and ultimate attainment in the pronunciation of a foreign language. Studies in Second Language Acquisition, 19/4: 447-465.

Casado y García (2000). Consideraciones didácticas sobre la enseñanza de lenguas extranjeras asistida por ordenador. Didáctica (Lengua y Literatura). 12: 67-89

Henning, G. (1973) Remembering foreign language vocabulary: Acoustic and semantic parameters. Language Learning, 23: 185-196

Krashen, S. (1981), Second Language Acquisition and Second Language Learning. Oxford, Pergamon.

Lenneberg, E.H. (1967). Biological Foundations of Language. New York, Wiley

Radford, A. et al (1999). Linguistics: An Introduction. Cambridge: Cambridge University Press.

Regil, L.:Laberintosdigitales. Artículo publicado en Internet, recuperado de la página http:/ webcache. googleusercontent.comsearch?q=cache:TMwC8DG JDzcJ:www.mexicandesign.com/revista/hipermedia. $\mathrm{htm}+$ hipermedia+y+pensamiento\&cd=10\&hl=es\&ct= clnk\&gl=co (25.10.2010)

Siemens, G. (2004). Conectivismo: Una teoría de aprendizaje para la era digital, Publicado bajo Licencia Creative Commons 2.5

Steinberg, D. (1996). An Introduction to Psycholingusitics. London and New York: Longman. 
Francisco Moreno Mosquera

\section{Anexo No.1}

\section{Formato de autorización de los participantes}

Quibdó, 24 de agosto de 2009

Apreciado padre de familia:

El Grupo de Investigación en Didáctica de los Idiomas Extranjeros de la Universidad Tecnológica del Chocó “Diego Luis Córdoba” se propone llevar a cabo un proyecto de investigación titulado Enseñanza del inglés a niños a través de la multimedia, en el cual involucraremos a niños con edades entre los 6 y los 10 años de edad.

Por lo anterior, muy comedidamente queremos solicitarle su autorización para que su hijo participe en el proyecto, como aprendiz del inglés. El niño deberá asistir a la UTCH (laboratorio de idiomas) por un período de tres meses los días lunes, miércoles y viernes en horas de la tarde. La Universidad se responsabiliza del transporte, y recogerá y entregará al menor en su casa

Si usted está de acuerdo en que su hijo haga parte del proyecto, le agradecemos firmar el formato adjunto.

Atentamente,

FRANCISCO MORENO MOSQUERA

Coordinador

Grupo de Investigación en Didáctica de los Idiomas Extranjeros

Universidad Tecnológica del Chocó

"Diego Luis Córdoba"

\section{THE AUTHOR}

FRANCISCO MORENO MOSQUERA holds an M.A. in English as a Foreign Language from University College of North Wales and has published a number of books and articles in national and international journals. Currently, he is a full time teacher at the Universidad Tecnológica del Chocó "Diego Luis Córdoba". 